\title{
Lamb Waves Topological Imaging of Multiple Blind Defects in an Isotropic Plate
}

\author{
Wen-Fa Zhu \\ School of Communication and Information Engineering, Shanghai University, Shanghai 200444, P.R. China. \\ School of Urban Railway Transportation, Shanghai University of Engineering Science, Shanghai 201620, P.R. \\ China.
}

\author{
Hai-Yan Zhang, Fan-Jie Liu, Meng-Yun Xu and Guo-Peng Fan \\ School of Communication and Information Engineering, Shanghai University, Shanghai 200444, P.R. China.
}

\begin{abstract}
Xiao-Dong Chai
School of Urban Railway Transportation, Shanghai University of Engineering Science, Shanghai 201620, P.R. China.
\end{abstract}

\author{
Kai-Liang Xu \\ Institut Langevin, ESPCI Paris, CNRS UMR 7587, INSERM U979, 17 rue Moreau, 75012 Paris, France.
}

\begin{abstract}
(Received 21 January 2018; accepted 20 June 2018)
The study investigates the feasibility of the Lamb wave topological imaging method for detecting multiple blindholes in an isotropic plate. The topological imaging method is performed based on the computations of two wave fields, a forward and an adjoint, in the defect-free reference medium using different emitting sources. The image is computed by multiplying the forward and adjoint wave fields together and integrating them over time or frequency. The interferences of multimode aliasing and the scattering effect can thus be eliminated at the defectfree positions with an improved image resolution. To investigate the physical mechanism, the refocusing process of the multimode Lamb waves at the defect positions is presented by a face-to-face comparison between the snapshots of the forward and adjoint wave fields using the finite element simulation. The Lamb wave topological imaging method is numerically and experimentally verified to identify multiple blind-holes in an isotropic aluminium plate. The results demonstrate that the topological imaging method enables the suppression of the sartefacts resulting from the mode conversion and achieve high-resolution imaging of the blind defects.
\end{abstract}

\section{INTRODUCTION}

The inverse scattering imaging has attracted considerable attentions in the biomedical engineering, industrial nondestructive testing and geophysics. ${ }^{1,2}$ Diffraction tomography, ${ }^{3}$ developed from inverse scattering algorithm, is capable of rapidly fulfilling the tomography imaging by using the Fast Fourier Transform (FFT) under the Born approximation assumption. However, the application of diffraction tomography is limited since Born approximation assumption is only applicable to the weak scattering case.

The topological optimization method has been applied to ultrasonic bulk wave imaging. ${ }^{4-8}$ The topological asymptote is applied and the defect imaging can be obtained without the prior assumptions of locations and shapes of defects. This method is derived from the shape optimization in mathematics. Early in 1994, Eschenauer et al. ${ }^{4}$ proposed the concept of topological gradient for topological optimization of mechanical structures. In 2001, Garreau et al. ${ }^{5}$ developed an asymptotic expansion in the context of linear elasticity for general functional and arbitrary shaped holes by using an adaptation of the adjoint method and a domain truncation technique. The proposed method was general and can be readily adapted to other linear partial differential equation and other types of boundary conditions. In 2004, Bonnet and Guzina ${ }^{6,7}$ extended the topological derivative to solve elastic wave inverse scattering problem. They illustrated the methodology enables to characterize the topological differentia towards the gradient-based imaging. In the field of ultrasonic nondestructive inspection, Gallego et al. ${ }^{8}$ conducted the inspection of crack and chamber defects using topological sensitivity boundary integral method.

Topological gradient method has been used to achieve a high resolution imaging. ${ }^{9,10}$ However, the method requires many iterations. Subsequently, the researchers began to explore the use of other fast imaging methods, for instance the time domain topological energy method proposed by Dominguez and Gibiat ${ }^{11}$ and frequency domain topological imaging by Rodriguez et al. ${ }^{12}$ Owing to the refocusing properties of the time-reversal ${ }^{13-15}$ for the scatterers, an accurate image could be readily obtained by solving two wave propagation problems in an homogeneous medium. Despite the iterative nature of the topological method, a single iteration leaded to an accurate image. ${ }^{11,12}$ Basing on the improvement of the topological imaging theory and algorithm, the experimental works are carried out successively. Tokmashev et al. ${ }^{16}$ has conducted the experimental investigation of elastic wave topological sensitivity for the aluminum plate with dual defects of a circular hole and a rectangular slit using a single longitudinal piezoelectric trans- 
ducer along the edges of the plate under varying transducer locations.

Rodriguez et al. ${ }^{17,18}$ recently combined the topological imaging method with the guided wave tomography, so-called guided wave topological imaging, which can provide the defect imaging in the isotropic and anisotropic plates. Existing studies have investigated the through-thickness defects basing on single mode excitation. However, the Lamb wave topological imaging for hidden asymmetric defects has not been reported yet. Furthermore, it has been widely analyzed that Lamb mode conversion can occur upon encountering the blind holes, generating the newly-converted modes apart from the reflection and transmission. ${ }^{19}$ It brings challenges for multimode scattering signal interpretation and leads to artifacts in the image. Nevertheless the impact of mode conversion for guided waves topological imaging has not been thoroughly discussed.

The present study focuses on the application of Lamb wave topological imaging with significant mode conversion. We considered the multiple blind holes in an isotropic plate, where significant mode conversion can be observed. In Section 2, the theory and the algorithm of topological imaging method are introduced. In Section 3, a finite element model is built to investigate the physical mechanism of topological method for the inspection of multiple blind holes. The experimental verification of the method is given in Sections 4.

\section{TOPOLOGICAL IMAGING THEORY}

As shown in Fig. 1, the medium containing the defects is called the inspected medium, $\Omega_{m}$. The defect-free medium, $\Omega_{0}$, which has the same properties as the inspected medium, is initialized as the reference medium. The process of gradual approach from $\Omega_{0}$ to $\Omega_{m}$ through insertion of a defect into $\Omega_{0}$ is named as the topological asymptote. The new medium obtained in each step is called the virtual modified medium, $\Omega_{\epsilon}$. The positive definite cost function $j\left(\Omega_{\epsilon}\right)$ is used to evaluate the difference of the displacement fields between the calculated and reference mediums.

$$
\begin{aligned}
j\left(\Omega_{\epsilon}\right)= & \frac{1}{2} \int_{R}\left(\iint_{(x, y) \in \Gamma_{m}}\left|u_{\epsilon}-u_{m}\right|^{2} \mathrm{~d} x \mathrm{~d} y\right) \mathrm{d} t= \\
& \frac{1}{2} \int_{R}\left(\int_{(x, 0) \in \Gamma_{m}}\left|u_{\epsilon}-u_{m}\right|^{2} \mathrm{~d} x\right) \mathrm{d} t,
\end{aligned}
$$

where $u_{\epsilon}$ represents the calculated displacement field at the boundary $\Gamma_{m}$ of the reference medium or the virtual modified medium, and $u_{m}$ is the measured displacement field obtained experimentally at the boundary $\Gamma_{m}$ of the inspected medium, $(x, y)$ denotes the coordinate in space $\Omega_{0}$ or $\Omega_{m}$, and $R$ is the duration time. Considering that a linear array is set along an edge of the inspected plate, $x$ defines the lateral positions of all array-elements, and $y=0$ in the study. The topological imaging can be obtained by retrieving an optimal solution after minimizing the cost function. Consequently, the virtual modified medium converges to the inspected medium. The variables, $j\left(\Omega_{0}\right)$ and $j\left(\Omega_{\epsilon}\right)$, represent the cost functions of the reference and virtual modified medium, respectively. The asymptotic expansion of the cost function is given by

$$
j\left(\Omega_{\epsilon}\right)=j\left(\Omega_{0}\right)+f(\epsilon) g(x, y)+o(f(\epsilon)),
$$

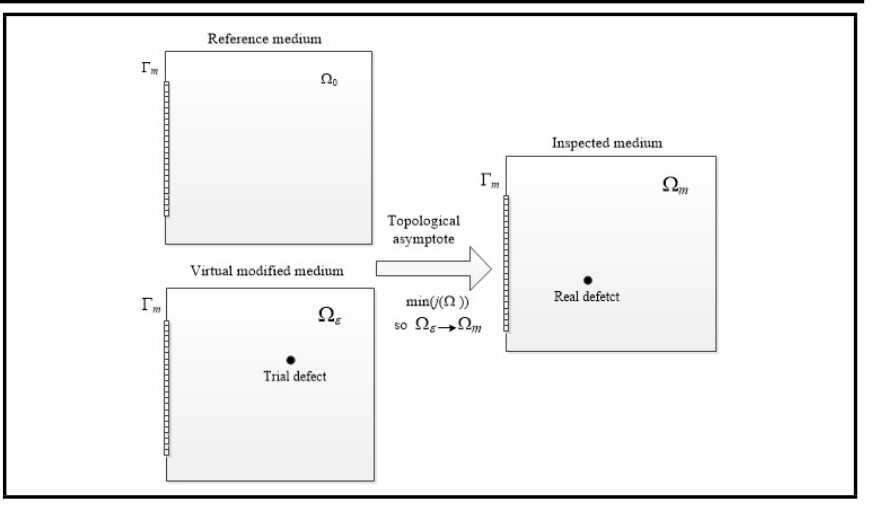

Figure 1. Principle of the topological asymptote

where $\epsilon$ stands for the defect parameter, $f(\epsilon)$ is the expression function of the defect and $o(f(\epsilon))$ represents the higher-order small term of $f(\epsilon)$. Ignoring the impact of the higher-order small terms, Eq. (3) can be written as

$$
g(x, y)=\frac{j\left(\Omega_{\epsilon}\right)-j\left(\Omega_{0}\right)}{f(\epsilon)},
$$

where $g(x, y)$ is so-called topological gradient. Dominguez gave the specific function expression of $g(x, y)$ and $f(\epsilon)$ using Dirichlet and Neumann types of boundary conditions under two-dimensional and three-dimensional models, respectively. ${ }^{9}$ Using the topological gradient $g(x, y)$ as the imaging function, the gradient value of each point in the imaging area is calculated. Thus, the topological gradient image can be obtained.

The topological asymptotic method, which takes the topological gradient as the imaging function, involves more iterations which, lead to relatively low computation efficiency. In this study, we choose an efficient topological energy imaging method. This method regards topological energy $g_{0}(x, y)$ as the imaging function by transforming the topological gradient to solve the solutions of forward problem and adjoint problems.

The imaging methodology is described as follows. First the analytical solutions of the forward problem leading to $U_{0}(x, y, t)$ in the reference medium $\Omega_{0}$ are calculated according to the Lamb wave propagation model, where the source is the numerical or experimental emission signal $e_{0}(t)$. Then, a measurement or simulation is performed on the reference and inspected medium that gives $u_{0}^{n}(t)$ and $u_{m}^{n}(t)$ on the array of transducers, respectively, where the superscript $n$ is the transducer number. The time reversed difference $u_{m}^{n}(t)-u_{0}^{n}(t)$ is then used as the source of the adjoint problem to compute $V_{0}(x, y, t)$ in the reference medium. Finally, the topological energy is determined and the image of the inspected medium is given. The topological imaging process using the time domain topological energy method is shown in Fig. 2.

The detailed description of the imaging process is as follows:

1. The forward problem is solved according to the Lamb wave propagation model in the reference medium $\Omega_{0}$. Let the exciting signal be $e_{0}(t)$, and the displacement field $U_{0}(x, y, t)$ at the imaging area $(x, y)$ is calculated using the analytical solution. ${ }^{20}$

$$
U_{0}(x, y, t)=F^{-1}\left\{F\left\{e_{0}(t)\right\} \exp \left(\frac{-i 2 \pi f d}{c_{p}(f)}\right)\right\},
$$




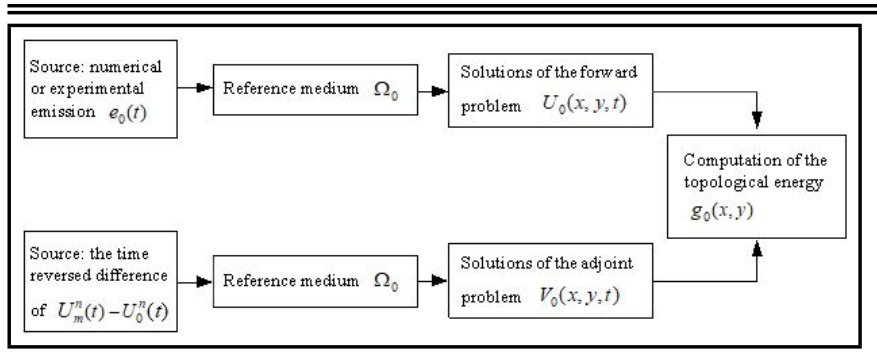

Figure 2. The process of topological imaging using the time domain topological energy method

where $F\{\bullet\}$ and $F^{-1}\{\bullet\}$ are the operators of the forward and inverse Fourier transform, respectively, $i$ is the imaginary symbol, and $c_{p}(f)$ is the phase velocity of the Lamb wave mode computed by using the Rayleigh-Lamb equation. ${ }^{21}$ The expression $d=\sqrt{\left(x-x_{0}\right)^{2}+\left(y-y_{0}\right)^{2}}$ represents the distance between the location $(x, y)$ and emitting source coordinate $\left(x_{0}, y_{0}\right)$.

2. The scattered Lamb wave signals are acquired by the experiment or simulation. Set a number of transducers on the boundaries $\Gamma_{m}$ of the reference medium $\Omega_{0}$ and inspected medium $\Omega_{m}$, respectively. Each of the transducers is used as the emitter and the rest are receivers. The variables $u_{0}^{n}(t)$ and $u_{m}^{n}(t)$ are the recorded response signals in the reference medium $\Omega_{0}$ and the inspected medium $\Omega_{m}$, respectively. The difference $h_{0}^{n}(t)=$ $u_{m}^{n}(t)-u_{0}^{n}(t)$ are the scattered Lamb wave signals.

3. The adjoint problem is solved according to the Lamb wave propagation model in the reference medium $\Omega_{0}$. Assume that at a low frequency-thickness product range there only exist two fundamental Lamb modes, symmetrical $S_{0}$ mode and asymmetrical $A_{0}$ mode. The signal $z_{0}^{n}(t)$ obtained by the time reversal of $h_{0}^{n}(t)$, is used as excitation. The adjoint displacement field $V_{0}(x, y, t)$ in the imaging area is calculated by

$$
\begin{aligned}
& V_{0}^{n}(x, y, t)=F^{-1}\left\{\alpha F\left\{z_{0}^{n}(t)\right\} \exp \left(\frac{-i 2 \pi f d}{c_{p}^{S_{0}}(f)}\right)\right\}+ \\
& F^{-1}\left\{\beta F\left\{z_{0}^{n}(t)\right\} \exp \left(\frac{-i 2 \pi f d}{c_{p}^{A_{0}}(f)}\right)\right\},
\end{aligned}
$$

where $\alpha, \beta, c_{p}^{S_{0}}(f), c_{p}^{A_{0}}(f)$ are propagating coefficient and phase velocities of $S_{0}$ and $A_{0}$ modes, respectively. Equation (6) takes into account the influence of mode conversion. A similar expression to Eq. (6) can be derived for more guided modes. According to the reciprocity principle, during the forward propagation, the time reversal signal $z_{0}^{n}(t)$ will refocus at the location of the defect. $^{22}$ Ignoring the mutual scattering influence among the defects, the focusing process of converted multimode signals at the defect location is depicted in Fig. 3. The identical mode signals $S_{0} / S_{0}$ and $A_{0} / A_{0}$ generated by $S_{0}$ and $A_{0}$ overlap at the defect location, which are called the main lobes. The signal $A_{0} / S_{0}$ denotes an $A_{0}$ mode converted from $S_{0}$ mode and $S_{0} / A_{0}$ denotes an $S_{0}$ mode converted from $A_{0}$ mode. The two modes are distributed on the two sides of the main lobes and their amplitudes are relatively smaller than that of the main lobes, called the side lobes.

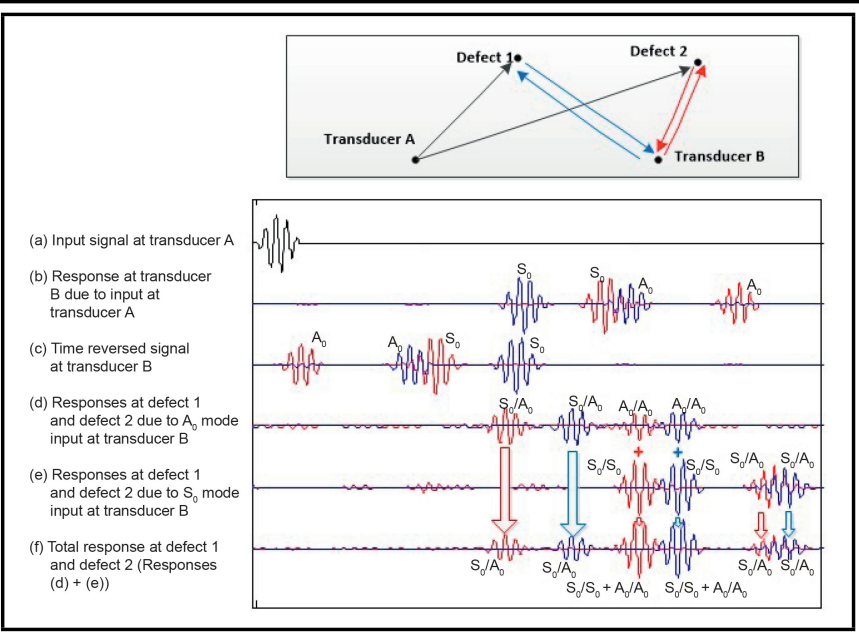

Figure 3. The focusing process of converted multi-mode signals at the defect locations. (Note: A0/S0 denotes A0 mode converted from S0 mode due to the interaction of the defect. $S_{0} / A_{0}, S_{0} / S_{0}$, and $A_{0} / A_{0}$ are similarly defined). The blue and red lines represent the scattering signals produced by defect 1 and 2 , respectively.

4. Using the time domain topological energy as the imaging function, the energy value at each point $(x, y)$ in the imaging area is computed. The topological energy is

$$
g_{0}(x, y)=\int_{0}^{T}\left\|U_{0}(x, y, t)\right\|^{2}\left\|V_{0}(x, y, t)\right\|^{2} \mathrm{~d} t
$$

where $T$ is the duration and $(x, y)$ is the coordinate location in the imaging area.

By introducing the forward displacement field and multiplying it with the adjoint displacement field, the topological energy imaging function can eliminate the interference of multimode overlap at the defect-free position. It enables focusing the energy on the defect location, which overcomes the disadvantage of other imaging methods that use only scattering signals and improves the resolution significantly.

(5) To further improve the image quality, the iteration procedure can be carried out by changing the emitting source position and repeating steps (1) through (4). Consolidating all the imaging results, a relatively high accuracy image can be obtained.

\section{SIMULATIONS}

\subsection{Mechanism of Topological Imaging}

To explain the refocusing physical mechanism of the multimode guided waves at defect locations, the snapshots of transient displacement field at the different moments were computed using the finite element simulations. The simulation was carried out by using the commercial software PZFlex. The defect image is reconstructed based on the topological imaging method. The numerical model of an isotropic aluminium plate is shown in Fig. 4. A $500 \times 500 \times 3 \mathrm{~mm}^{3}$ aluminium plate was meshed using hexahedral elements with the element size of $1.3 \times 1.3 \times 0.5 \mathrm{~mm}^{3}$. The total number of elements was $385 \times 385 \times 6=889350$. Absorbing boundary conditions were set to prevent the reflections from the edges. The imaging region of interest is located at the centre of the model with a size of $100 \times 100 \mathrm{~mm}^{2}$. The material parameters of the aluminium are Young modulus $69 \mathrm{GPa}$, density of $2.69 \mathrm{~g} / \mathrm{cm}^{3}$, and 


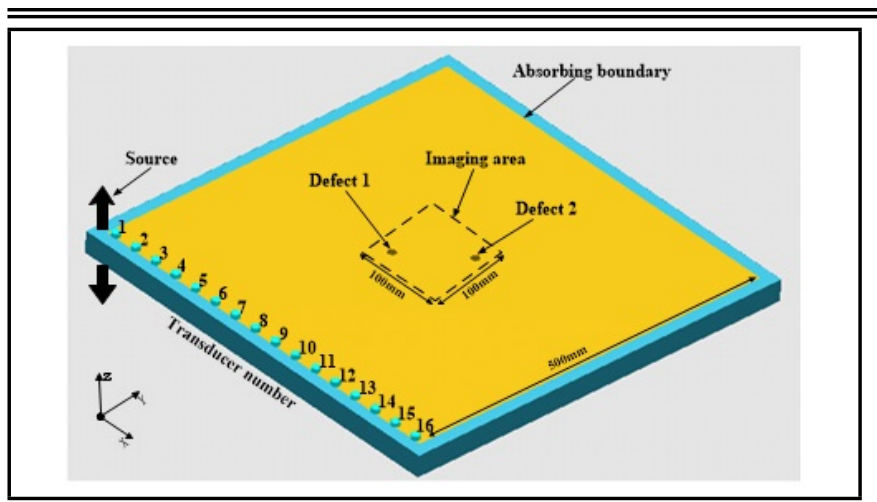

Figure 4. Schematic diagrams of the finite element model.

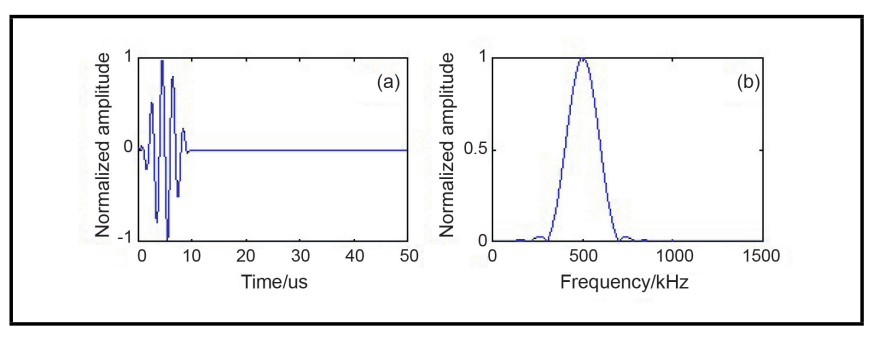

Figure 5. Emitting signal (a) waveform and (b) spectrum.

Poisson's ratio 0.35 . Time duration is set as $200 \mu$ s with a sampling interval of $84.6 \mathrm{~ns}$. Sixteen transducers are arranged on the left side of the model. The interval distance between each two transducers is $30 \mathrm{~mm}$, and their diameter is $2.5 \mathrm{~mm}$. Two circular blind holes with a diameter $10 \mathrm{~mm}$ locate at $(-30 \mathrm{~mm}$, $-30 \mathrm{~mm}$ ) and $(30 \mathrm{~mm}, 3 \mathrm{~mm})$. To model the partial-thickness defects, the holes are asymmetric to the mid-plane of the plate and are $1 \mathrm{~mm}$ in depth. The mid-plane refers to the horizontal section at $1 / 2$ plate thickness, which is perpendicular to the plate thickness direction. As shown in Fig. 5, the emitting $e_{0}(t)$ is a five-cycle Hanning-windowed sinusoidal signal with a $500 \mathrm{kHz}$ centre frequency. At this frequency, the wavelength $\lambda$ of $S_{0}$ mode is $10.6 \mathrm{~mm}$ and close to the defect diameter, which denotes the challenges of hide-defect imaging.

In the numerical example, the plate is excited by transducer 1 and the backscattering signal is recorded by all transducers. The source is applied symmetrically on the top and bottom surfaces of the plate to selectively excite the symmetric $S_{0}$ mode. As a result, the $S_{0}$ mode is emitted at frequencythickness product $1.5 \mathrm{MHz} \cdot \mathrm{mm}$ according to the Lamb wave dispersion curves.

Figure 6 depicts the transient displacement field of $S_{0}$ mode at a different time. The scattering and mode conversion can be observed. At $1.5 \mathrm{MHz} \cdot \mathrm{mm}$ frequency-thickness product, the group velocity of $A_{0}$ mode is smaller than that of $S_{0}$ mode. As shown in Figs. 6c and 6d, the two different wave-fronts of $S_{0}$ and $A_{0}$ modes can be clearly distinguished.

Figure 7 shows the focusing process of the forward and adjoint displacement fields. Lamb wave scattering signals $h_{0}^{n}(t)=u_{m}^{n}(t)-u_{0}^{n}(t)(n=1 \sim 16)$ are shown in Fig. 7a. It can be seen from these waveforms that the signals scattered by the two defects include multiple modes. To obtain the adjoint wave field, the signals in Fig. 7a were then time-reversed (see Fig. 7b) and regarded as the emissions in adjoint problem, and the corresponding adjoint displacement field can be computed according to Eq. (6). As shown in Figs. 7c and 7d, in the forward and adjoint displacement fields, the wave-packets arrive
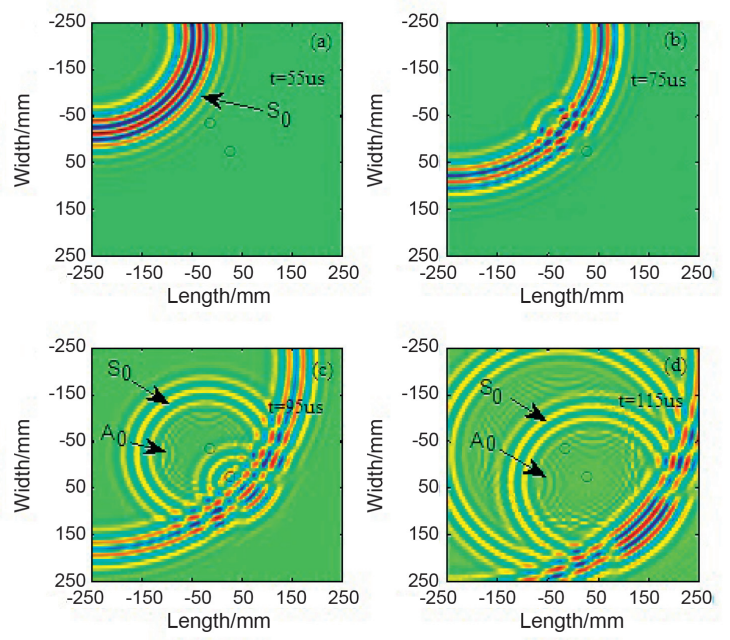

Figure 6. Transient displacement fields of Lamb waves at the different moments: (a) when $t=55 \mu \mathrm{s}$, before wave-front encountering the defects; (b) when $t=75 \mu \mathrm{s}$, it is passing through defect 1 ; (c) when $t=95 \mu \mathrm{s}$, it is passing through defect 2 ; (d) when $t=115 \mu \mathrm{s}$, it is departing from the two defects.
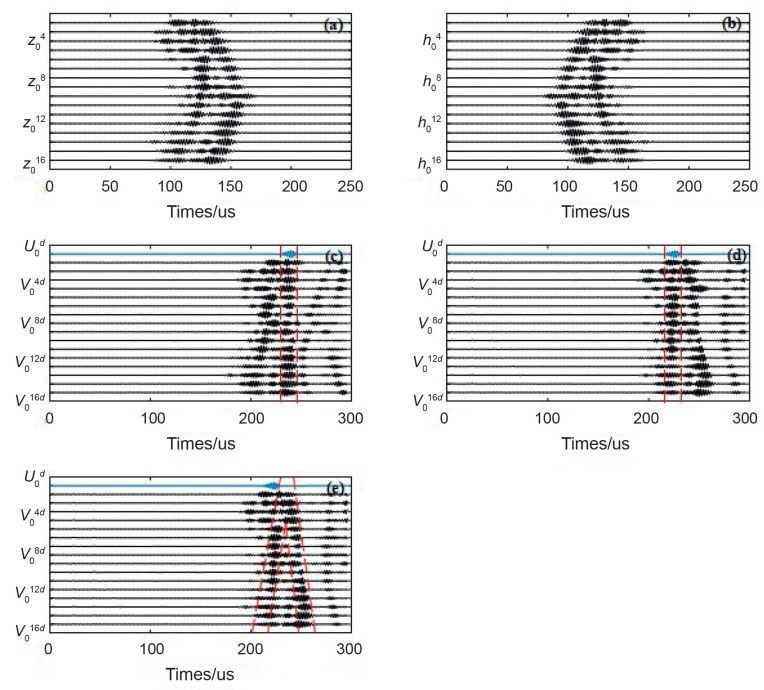

Figure 7. The simulated focusing process of the forward and adjoint displacement fields: (a) Scattering signals received by each transducer; (b) $z_{0}^{n}(t)$ are the time-reversed waveforms of signals in figure (a). According to Eq. (6), $z_{0}^{n}(t)$ are used as emissions to compute adjoint fields $V_{0}^{n}(t)$; Fig. 6c-e show the adjoint-field signals obtained at (c) defect 1 ; (d) defect 2; (e) a defectfree position $(-30 \mathrm{~mm}, 30 \mathrm{~mm}) . U_{0}^{d}(t)$ is the signal in the forward problem. $V_{0}^{n} d(t)$ is the adjoint-field signal and the superscript $d$ represents defect. $U_{0}^{h}(t)$ and $V_{0}^{n} h(t)$ are the signals at the defect-free location in the forward and adjoint displacement fields, respectively.

at the defect position at the same time. The adjoint field signals at the defect-free location $(-30 \mathrm{~mm}, 30 \mathrm{~mm})$ are shown in Fig. 7e. Different from the waveforms of the adjoint field computed using the defect coordinates (see Figs. 7c-7d), the wave-packets arrived at the array element with different time and no-focusing effect can be observed in Fig. 7e.

Figure 8 shows the focusing process of the forward and adjoint problems at the two defect locations using the snapshots of the transient displacement fields. Figures $8 \mathrm{a}-8 \mathrm{~d}$ in the left column illustrate the propagating process of acoustic waves 


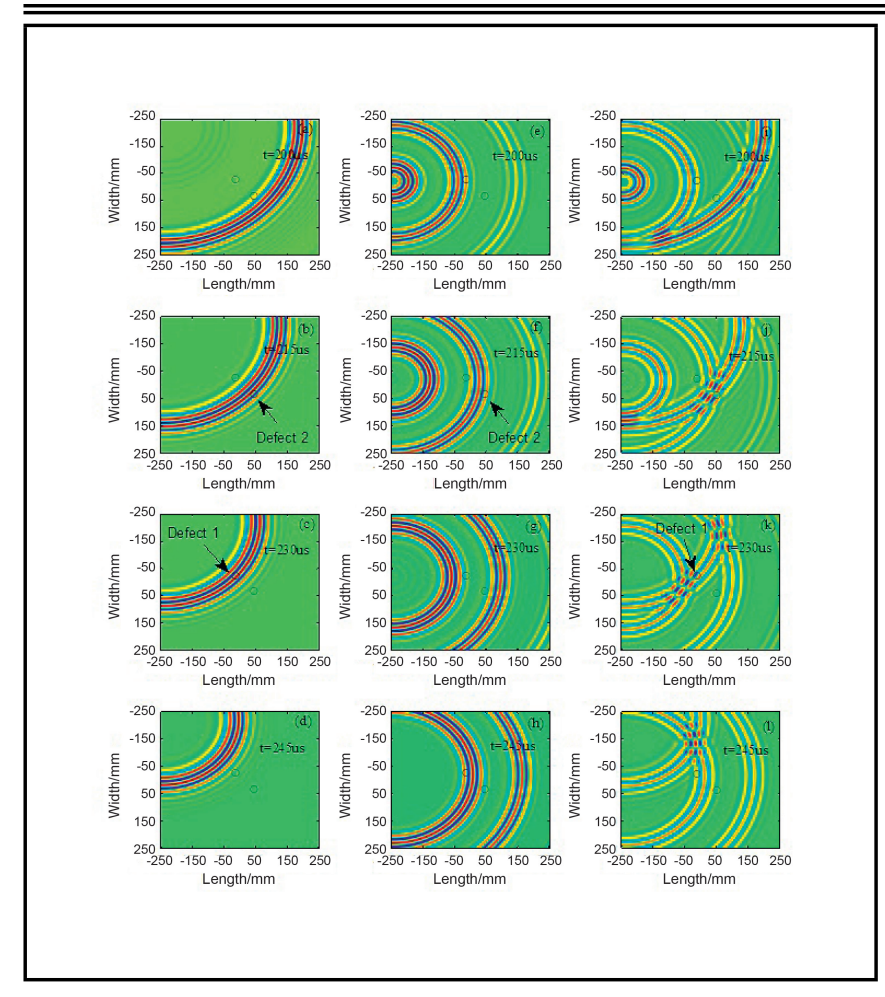

Figure 8. The snapshots of transient displacement fields in the forward and adjoint problems: (a)-(d) forward problem; (e)-(h) adjoint problem; (i-1) the product of the corresponding two displacement fields. Please note that in Figs. $7 \mathrm{a}-\mathrm{d}$, the wave seems to propagate backward because the time is reversed with $t=T-t_{p}$, where $t_{p}$ is the moment at which the source $e_{0}(t)$ propagates to the location $p(x, y)$ and $T$ is the total propagating time.

in the forward problem, in which the emitting signal $e_{0}(t)$ is applied on transducer 1 with the coordinates $(-225 \mathrm{~mm}$, $-247 \mathrm{~mm}$ ). Figures $8 \mathrm{e}-8 \mathrm{f}$ illustrate the propagating process of acoustic waves in the adjoint problem, in which the emitting signal $z_{0}^{7}(t)$ in Fig. $7 \mathrm{~b}$ is applied on transducer 7 with the coordinates $(-45 \mathrm{~mm},-247 \mathrm{~mm})$. It can be seen from Figs. $8 \mathrm{~b}$ and $8 \mathrm{f}$ that the displacement fields of the forward and adjoint problems arrive at the defect 2 simultaneously when $t=215$ $\mu \mathrm{s}$. In Figs. $8 \mathrm{c}$ and $8 \mathrm{~g}$, the two displacement fields arrive at the defect 1 simultaneously when $t=230 \mu$ s. Figures $8 \mathrm{i}-1$ illustrate the product of the corresponding two displacement fields. Similar to the waveforms shown in Fig. 7, the defect focusing imaging can be seen from the snapshots of the transient displacement fields.

\subsection{Topological Imaging}

Figure 9 illustrates the imaging results and the intensity profile views in length and width direction using the methods of delay and sum (DAS) and topological imaging under the same emission and reception condition, respectively. In the profile view, the parameters of the $-2.5 \mathrm{~dB}$ main lobe width $\omega$ are used to quantify the imaging resolution. High resolution corresponds to small main lobe width. It can be seen that the traditional DAS method has a large defect size with a high ambiguity of defect location. The $-2.5 \mathrm{~dB}$ main lobe widths of the DAS method and the topological method are $\omega=\max \omega_{1} \sim \omega_{4} \approx 30 \mathrm{~mm}$ and $\omega=\max \omega_{1} \sim \omega_{4} \approx 10$ $\mathrm{mm}$, respectively. As the defect radius was $10 \mathrm{~mm}$, the imaging defect size of the topological method is more accurate than the DAS method. Both methods enable to provide correct defect locations in simulation.

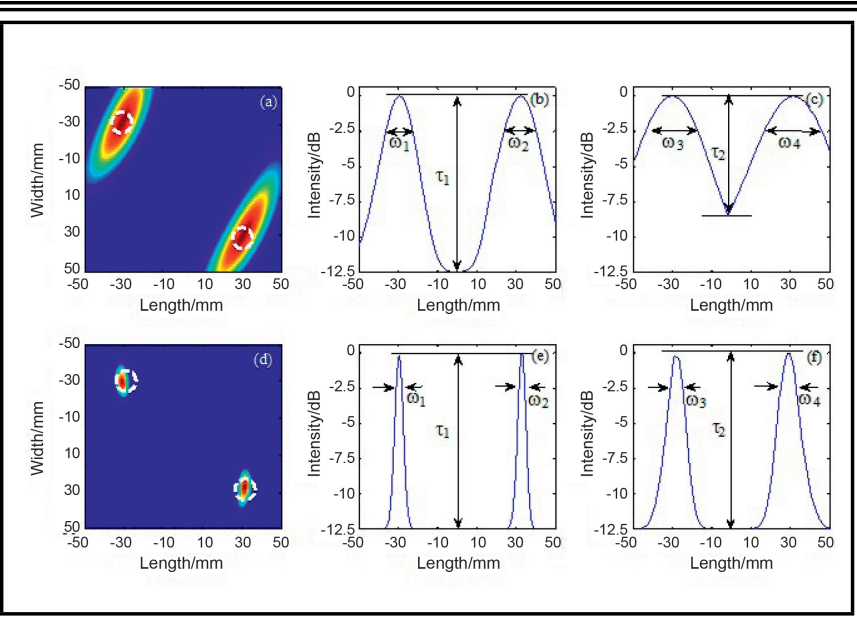

Figure 9. Simulation of two defects: DAS imaging methods (a) defect imaging; (b) width profile view; (c) length profile view of DAS; topological imaging method (d) defect imaging; (e) width profile view; (f) length profile view.

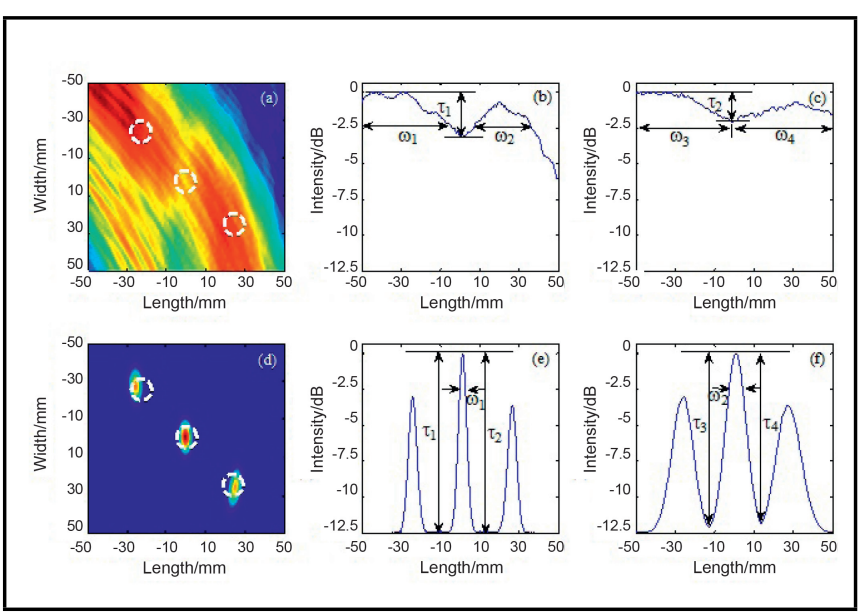

Figure 10. Simulation of three defects: DAS imaging methods (a) imaging, (b) width profile view, (c) length profile view; topological imaging methods (d) defect imaging; (e) width profile view; (f) length profile view.

Figure 10 depicts the imaging results of three blind holes with a diameter of $10 \mathrm{~mm}$ and a defect depth of $1 \mathrm{~mm}$. The positions of the three blind holes are $(-25 \mathrm{~mm},-25 \mathrm{~mm})$, (0 mm, $0 \mathrm{~mm})$, and $(25 \mathrm{~mm}, 25 \mathrm{~mm})$, respectively. As shown in Figs. 10b-c, DAS results are obtained with $\omega>50 \mathrm{~mm}$ and $\tau>-2.5 \mathrm{~dB}$, though failing to localize the three defects individually, it can be seen in Figs. 10d-f that the topological method still can distinguish the three defects with $\omega=$ $\max \omega_{1} \sim \omega_{4} \approx 8 \mathrm{~mm}$ and $\tau<-2.5 \mathrm{~dB}$.

\section{EXPERMENTAL VERIFICATION}

To verify the reliability of topological imaging, the experiment was performed in a $3 \mathrm{~mm}$-thickness aluminium plate. Two holes were drilled partly through the plate thickness with diameters of $4 \mathrm{~mm}$. Figure 11a shows the position of the drilled holes on the plate. Measurements were carried out using an M2M MultiX LF system. A 16-element linear array (Shantou Ultrasonic Electronics Co. LTD Guangdong, China) was used. The array is composed of 16 transducers whose central frequency is $500 \mathrm{kHz}$. The pitch size is $2 \mathrm{~mm}$ and thus the whole array is $32 \mathrm{~mm}$ long. The transducer width is $1.8 \mathrm{~mm}$ and the height is $15 \mathrm{~mm}$. Similar to previous literatures, the array is coupled to the plate on the edge, so that it is sensitive to the 


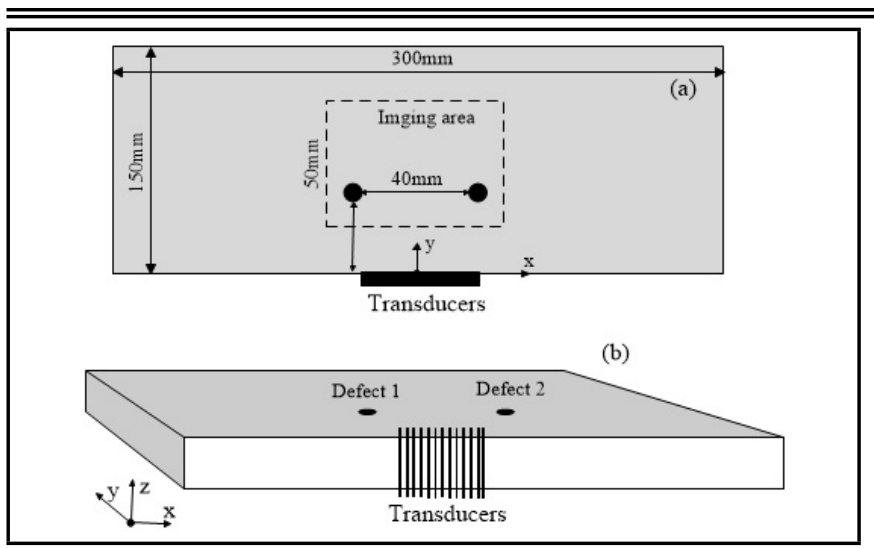

Figure 11. Experimental configuration: (a) vertical view; (b) front view

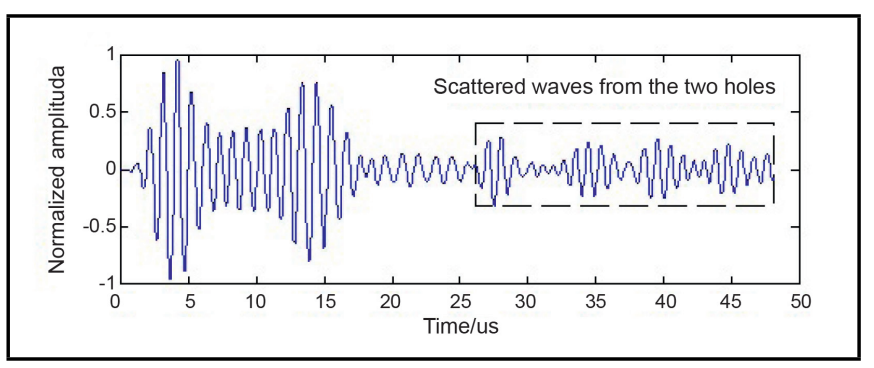

Figure 12. Typical measured signal.

in-plane motion. ${ }^{17,18}$ As shown in Fig. 11b, since the transducer height is larger the plate thickness, the transducers cover entire thickness of the plate. Considering the $3 \mathrm{~mm}$ thickness of the plate, the corresponding frequency-thickness product is $1.5 \mathrm{MHz} \cdot \mathrm{mm}$.

The source is successively applied on the transducers along the $\mathrm{x}$-axis at the plane $y=0$. The emitting signal is a five-cycle Gauss-windowed sinusoidal signal with $0.5 \mathrm{MHz}$ central frequency. One of the transducers is used as emitter and all transducers (including the emitter itself) record the scattering Lamb wave signals. Using the single-emitter and multi-receiver measurement, a total of 256 channels signals are recorded by the linear-array. A typical measured signal is shown in Fig. 12. The excitation wave-packets are followed by the scattered waves from the two holes. Because of the mode conversion between $S_{0}$ and $A_{0}$ modes at two blind holes, multi-scattering wave-packets can be observed in the recorded signals. However, as shown in Figs. 13a and 13b, the forward and adjoint displacement fields focus on the two defect locations with an identical arrival time; on the contrary, unfocused results can be seen in Fig. 13c in the non-defect region.

The imaging results of the topological and DAS methods are presented in Fig. 14. In Fig. 14a, the two defects can be clearly distinguished from the topological imaging result without significant artifacts, while the DAS result in Fig. 14b presents obvious sidelobes and ring artifacts. However, because of the influence of the boundary reflection and the dispersion of multimode guided waves, the imaging quality is not as good as in the simulation.

\section{DISCUSSION AND CONCLUSIONS}

This work investigates the applicability of the Lamb wave topological imaging method for the inspection of multiple blind-hole defects in an isotropic aluminium plate. Based on
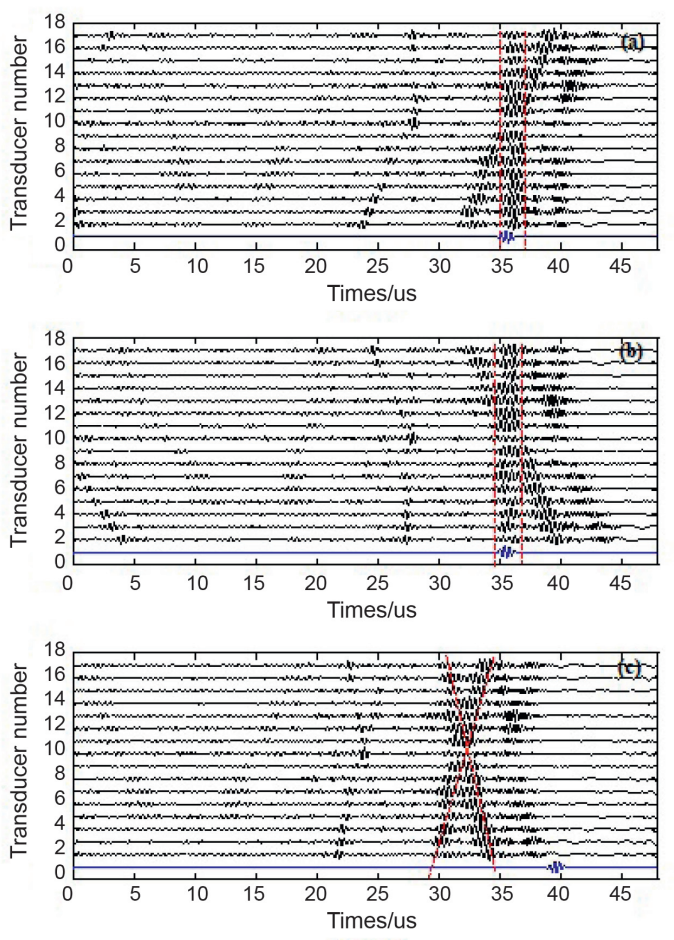

Figure 13. The focusing of mode conversion signals and multiple scattering signals from two defect in the experiment. (a) Focusing at defect 1; (b) focusing at defect 2; (c) unfocusing at non-defective position.

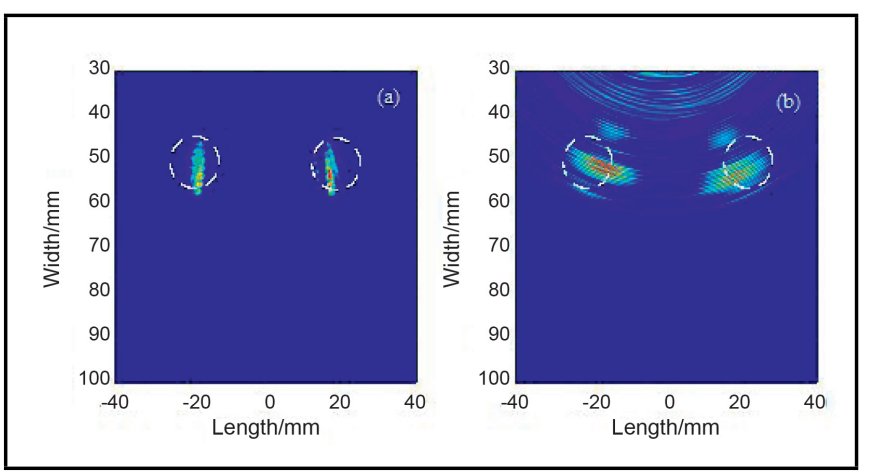

Figure 14. Experimental results for defect imaging of two holes in a $3 \mathrm{~mm}-$ thickness aluminum plate: (a) topological method; (b) DAS method.

the Lamb wave propagation model, the solutions displacement fields in the forward and adjoint problems are solved using the different emitting source in the reference medium. The multimode Lamb wave imaging mechanism is explained using a face-to-face comparison between the snapshots of the forward and adjoint transient displacement fields. Both the numerical and the experimental results are presented for multiple blind-hole defects to verify the efficiency of the proposed method. The mode conversion is modelled in the adjoint problem, which enables the suppression of the artefacts in the defect image caused by the converted modes.

Compared with the classical DAS method, topological imaging enables refocusing the mode energy at the defect-free position, so that the artefacts can be suppressed with a significant contrast enhancement for the defect imaging with more accurate defect evaluation and localization. Taking advantage of the proposed multimode strategy for the adjoint field computation, the improved topological imaging method can overcome the artefacts that resulted from the mode conversion and 
multi-scattering, and further improve the defect imaging. Generally speaking, the topological method is able to provide high precision results for defect imaging with the same magnitude of computation time as the DAS method.

\section{ACKNOWLEDGEMENTS}

This work was supported by the National Natural Science Foundation of China under Grants Nos. 11674214, 11474195, 51478258, and Key Technology R\&D Project of Shanghai Committee of Science and Technology under Grant No.16030501400.

\section{REFERENCES}

1 Castaings, M. SH ultrasonic guided waves for the evaluation of interfacial adhesion, Ultrasonics, 54 (7), 1760-1775, (2014). https://dx.doi.org/10.1016/j.ultras.2014.03.002.

2 Mao, J., Leng, T., Zhuang, Z. Y., and Wang, X. M. Efficient acceleration for total focusing method based on advanced parallel computing in FPGA, International Journal of Acoustics and Vibration, 22 (4), 536-540, (2017). https://dx.doi.org/10.20855/ijav.2017.22.4500.

3 Rohde, A. H., Vedit, M., Rose, L. R. F., and Homer, J. A computer simulation study of imaging flexural inhomogeneities using plate-wave diffraction tomography, Ultrasonics, 48 (1), 6-15, (2008). https://dx.doi.org/10.1016/j.ultras.2007.09.002.

4 Eschenauer, H. A., Kobelev, V. V., and Schumacher, A. Bubble method for topology and shape optimization of structures, J. Struct. Optim., 8 (1), 42-51, (1994). https://dx.doi.org/10.1007/BF01742933.

5 Garreau, S., Guillaume, P., and Masmoudi, M. The topological asymptotic for PDE systems: The elasticity case, SIAM J. Control Opim., 39 (6), 1756-1778, (2001). https://dx.doi.org/10.1137/S0363012900369538.

6 Bonnet, M., and Guzina, B. B. Sounding of finite solid bodies by way of topological derivative, International Journal for Numerical Methods in Engineering, 61 (13), 23442373, (2004). https://dx.doi.org/10.1002/nme.1153.

7 Guzina, B., and Bonnet, M. Topological derivative for the inverse scattering of elastic waves, Quarterly Journal of Mechanics and Applied Mathematics, 57 (5), 161-179, (2004). https://dx.doi.org/10.1093/qjmam/57.2.161.

8 Gallego, R., and Rus, G. Identification of cracks and cavities using the topological sensitivity boundary integral equation, Computational Mechanics,33 (2), 154-163, (2004). https://dx.doi.org/10.1007/S00466-003-0514-4.

9 Dominguez, N., Gibiat, V., and Esquerre, Y. Time domain topological gradient and time reversal analogy: an inverse method for ultrasonic target detection, Wave Motion, 42 (1), 31-52, (2005). https://dx.doi.org/10.1016/j.wavemoti.2004.09.005.
10 Yuan, H., Bracq, G., and Liu, Q. Inverse acoustic scattering by solid obstacle: topological sensitivity and its preliminary application, Inverse Problems in Science and Engineering, 24 (1), 92-126, (2016). https://dx.doi.org/10.1080/17415977.2015.1017483.

11 Dominguez, N., and Gibiat, V. Non-destructive imaging using the time domain topological energy method, Ultrasonics, 50 (3), 367-372, (2010). https://dx.doi.org/10.106/j.ultras.2009.08.014.

12 Rodriguez, S., Sahuguet, P., Gibiat, V., and Jacob, X. Fast topological imaging, Ultrasonics, 52 (8), 1010-1018, (2012). https://dx.doi.org/10.1016/j.ultras.2012.08.002.

13 Devaney A. J. Time reversal imaging of obscured targets from multistatic data, IEEE Trans. Anten. Propag., 53 (5), 1600-1610, (2005).https://dx.doi.org/doi.org/ 10.1109/TAP.2005.846723.

14 Ing R., Fink M. Time-reversed Lamb waves. IEEE Trans. Ultrason., Ferroelect., Freq. Contr., 45 (4), 1032-1043, (1998). https://dx.doi.org/10.1109/58.710586.

15 Wang C. H., Rose J. T., Chang F. K. A synthetic time-reversal imaging method for structural health monitoring. Smart Mater. Struct., 13 (2), 415-423, (2004) https://dx.doi.org/10.1088/0964-1726/13/2/020.

16 Tokmashev, R., Tixier, A., and Guzina, B., B. Experimental validation of the topological sensitivity approach to elasticwave imaging, Inverse Problems, 29 (12), 1-25, (2013). https://dx.doi.org/10.1088/0266-5611/29/12/125005.

17 Rodriguez, S., Deschamps, M., Castaings, M., and Ducasse, E. Guided wave topological imaging of isotropic plates, Ultrasonics, 54 (7), 1880-1890, (2014). https://dx.doi.org/10.1016/j.ultras.2013.10.001.

18 Rodriguez, S., Castaings, M., Deschamps, M., and Ducasses, E. Topological imaging of defects in anisotropic plates. $7^{\text {th }}$ European Workshop on Structural Health Monitoring, Nantes, France, (2014). http://www.ndt.net/article/ewshm2014/papers/0384.pdf

19 Xu, K. L., Ta, D., Su, Z., Wang, W., Transmission analysis of ultrasonic Lamb mode conversion in a plate with partialthickness notch, Ultrasonics, 54 (1), 395-401, (2014). https://dx.doi.org/10.1016/j.ultras.2013.07.011.

20 Ross, M. L., and Jennifer, E. M. Model-based imaging of damage with Lamb waves via sparse reconstruction, J. Acoust. Soc. Am., 133 (3), 1525-1534, (2013). https://dx.doi.org/10.1121/1.4788984.

21 Balvantín, A. J., Diosdado-De-la-Peña, Limon-Leyva, P., A., and Hernández-RodrÍguez, E. Study of guided wave propagation on a plate between two solid bodies with imperfect contact conditions, Ultrasonics, 83, 137-145, (2018). https://dx.doi.org/10.1016/j.ultras.2017.06.003.

22 Park, H. W., Kim, S. B., and Sohn, H. Understanding a time reversal process in Lamb wave propagation, Wave Motion, 46, 451-467, (2009). https://dx.doi.org/10.1016/j.wavemoti.2009.04.004. 